\title{
MiR-558 inhibits odontogenic differentiation of human periodontal ligament fibroblasts by negatively regulating Jagged-1/Notch signaling pathway
}

\author{
Shengzhang Song ${ }^{1}$, Zhuanjun Yan ${ }^{1}$, Wangxi Wu ${ }^{2 *}$ \\ ${ }^{1}$ Department of Orthodontics, Qinghai University Affiliated Hospital, Xining City, Qinghai Province 810001, ${ }^{2}$ Department of Oral \\ Restoration, Guangdong Stomatological Hospital, Guangzhou City 510280, China
}

*For correspondence: Email: hongtaoli8@163.com; Tel: +86-0971-6162865

Sent for review: 8 February 2021

Revised accepted: 28 May 2021

\begin{abstract}
Purpose: To investigate the potential effect and explore the underlying mechanism of action of miR-558 in the odontogenic differentiation of human periodontal ligament fibroblasts (hPLFs).

Methods: Human periodontal ligament fibroblasts were treated with osteogenic induction medium to induce odontogenic differentiation. The efficiency of odontogenic differentiation was determined using Alizarin red staining and alkaline phosphatase (ALP) activity assays. The expression levels of osteogenic markers, including Osterix and Runx2, were determined by Western blotting, while mRNA levels of Jagged-1, HES1 and HEY1 were assessed by quantitative real-time polymerase chain reaction (qRT-PCR).

Results: MiR-558 was down-regulated during the odontogenic differentiation of hPLFs. Alizarin red staining and ALP activity data indicate that miR-558 significantly inhibited the odontogenic differentiation of hPLFs. Osterix and Runx2 expression levels were significantly decreased in the miR-558 mimic group but significantly increased in miR-558 inhibitor group compared with those in NC group $(p<0.01)$. Moreover, miR-558 regulated Notch signaling by targeting Jagged-1, while Jagged-1 knockdown suppressed the odontogenic differentiation of hPLFs.

Conclusion: MiR-558 inhibits the odontogenic differentiation of human periodontal ligament fibroblasts by negatively regulating Jagged-1/Notch signaling pathway, and thus, could serve as a potential target to regulate the odontogenic differentiation of hPLFS.
\end{abstract}

Keywords: MiR-558, Odontogenic differentiation, Jagged-1, Notch signaling pathway, Human periodontal ligament fibroblasts

\begin{abstract}
This is an Open Access article that uses a fund-ing model which does not charge readers or their institutions for access and distributed under the terms of the Creative Commons Attribution License (http://creativecommons.org/licenses/by/4.0) and the Budapest Open Access Initiative (http://www.budapestopenaccessinitiative.org/read), which permit unrestricted use, distribution, and
\end{abstract} reproduction in any medium, provided the original work is properly credited.

Tropical Journal of Pharmaceutical Research is indexed by Science Citation Index (SciSearch), Scopus, International Pharmaceutical Abstract, Chemical Abstracts, Embase, Index Copernicus, EBSCO, African Index Medicus, JournalSeek, Journal Citation Reports/Science Edition, Directory of Open Access Journals (DOAJ), African Journal Online, Bioline International, Open-J-Gate and Pharmacy Abstracts

\section{INTRODUCTION}

The periodontal ligament is the connective tissue embedded between the tooth root and inner wall of the alveolar socket bone, anchoring the teeth in the jawbone, and buffering mechanical stress during chewing [1]. Previous studies have shown that human periodontal ligament stem cells are a population of multipotent stem cells with a potential to differentiate into osteocytes, chondrocytes and adipocytes [1]. Human periodontal ligament fibroblasts (hPLFs) 
synthesize and secrete collagen fibers to maintain the main morphology and function of the periodontal ligament [1]. In addition, hPLFs have a potential for osteogenic differentiation that contributes to periodontal remolding [2]. The present study aimed to investigate the regulation mechanism of hPLFs in odontogenic differentiation during periodontal remolding.

MicroRNAs (miRNAs) are small non-coding RNA that regulate gene expression through posttranscriptional inhibition of mRNA translation and play an important role in various cellular processes [3]. MiRNAs not only play an important regulatory role in the self-renewal and differentiation of stem cells but also maintain the undifferentiated state of stem cells [3]. The odontogenic differentiation of pulp stem cells is a key step in pulp regeneration [4]. MicroRNAs play an important regulatory role in the odontogenic differentiation of stem cells [5]. MiR$27 a-5 p$ is significantly increased during the odontogenic differentiation of dental pulp stem cells and promotes odontogenic differentiation by inhibiting LTBP1, and miR-143-3p is also involved in regulating odontogenic differentiation [5]. Moreover, a previous study demonstrated that miR-675 promotes osteogenic differentiation by targeting the expression of distal-less homeobox [6]. However, miR-508-5p inhibits osteogenic differentiation because of the decreased expression of the target GPNMB [7]. We found that the expression of miR-558 in hPLFs is down-regulated during osteogenic differentiation; however, the role and potential mechanism of miR-558 in osteogenic differentiation have not yet been reported. Therefore, this study aimed to investigate the potential effect and explore the underlying mechanism of miR-558 in the odontogenic differentiation of human periodontal ligament fibroblasts.

\section{EXPERIMENTAL}

\section{Cell culture and transfection}

Human embryonic kidney 293T (HEK-293T) cells and hPLFs cells were used in the current study.
HEK-293T cells were obtained from the Type Culture Collection of the Chinese Academy of Sciences. Human periodontal ligament fibroblasts were purchased from ScienCell (California, USA). Human periodontal ligament fibroblasts were transfected with an miR-558 mimic or an miR-558 inhibitor using FuGENE (Roche Molecular Biochemicals). The knockdown of Jagged-1 was performed by transfection with short hairpin RNA (shRNA) targeting Jagged-1. The sequences of shRNA used in this study were from a previous report [8].

\section{Real-time polymerase chain reaction (qRT- PCR)}

Total RNA was extracted using TRIzol reagent (Invitrogen), and $1 \mu \mathrm{g}$ RNA was transcribed into cDNA using a reverse transcription kit (Takara). $\mathrm{PCR}$ reactions were performed using $2 \times \mathrm{SYBR}$ mix (Takara). The primers for miR-558 and U6 snRNA were designed and purchased from Guangzhou RiboBio Co., Ltd (Guangzhou, China). The primer pairs for qRT-PCR are shown in Table 1.

\section{Western blotting}

Samples were harvested using RIPA buffer (Beyotime, Shanghai, China). Total protein samples were separated by $8 \%$ SDS-PAGE and transferred to polyvinylidene fluoride (PVDF) membranes. The primary antibodies used were all purchased from Abcam and primarily contained Jagged-1 (ab7771; 1:1000; abcam, MA, USA), Osterix (ab209484; 1:1000; Abcam), Runx2 (ab192256; 1:1000; Abcam) and $\beta$-actin (ab8226; 1:1000; Abcam).

\section{Osteoblastic differentiation study}

Human periodontal ligament fibroblasts were treated with osteogenic induction medium containing $100 \mathrm{nM}$ dexamethasone (SigmaAldrich, St. Louis, MO, USA), $50 \mu \mathrm{g} / \mathrm{mL}$ of ascorbic acid (Sigma-Aldrich), and $10 \mathrm{mM} \beta$ glycerophosphate (Sigma-Aldrich). On days 0, 1, 2, 4, 7 and 14, cell samples were collected for further analysis.

Table 1: Primers used for qRT-PCR

\begin{tabular}{lll}
\hline Gene & \multicolumn{1}{c}{ Forward primer (5'-3') } & \multicolumn{1}{c}{ Reverse primer (5'-3') } \\
\hline Jagged-1 & AATGGCTACCGGTGTGTCTG & CCCATGGTGATGCAAGGTCT \\
HES1 & CTGAGCACAGACCCAAGTGT & GAGTGCGCACCTCGGTATTA \\
HEY1 & TAATTGAGAAGCGCCGACGA & TGCTCCATTACCTGCTTCTC \\
GAPDH & GAAAGCCTGCCGGTGACTAA & AGGAAAAGCATCACCCGGAG \\
\hline
\end{tabular}




\section{Alizarin red staining}

Mineralized nodules were stained using $1 \%$ Alizarin red S (Sigma-Aldrich). On day 14 after odontogenic differentiation induction, the cells were fixed with $4 \%$ paraformaldehyde at room temperature for $30 \mathrm{~min}$ and $1 \%$ Alizarin red $\mathrm{S}$ solution was then added. After incubation for 30 min, the cells were washed with $\mathrm{ddH}_{2} \mathrm{O}$ to remove unbound dye and photographed.

\section{Alkaline phosphatase (ALP) staining}

Human periodontal ligament fibroblasts were treated with osteogenic induction medium for 7 days. Cell samples were then harvested, and ALP activity was measured using a commercial kit (P0321S, Beyotime) according to the manufacturer's instructions.

\section{Dual-luciferase reporter assay}

Human embryonic kidney 293T cells were used for the dual-luciferase reporter assay as previously described[9]. Briefly, HEK-293T cells were co-transfected with the firefly-tagged pGL3 promoter luciferase vector (Promega) and the Renilla control luciferase vector (Promega). Forty-eight hours later, luciferase activities were determined, and the results were presented as the ratio of firefly/Renilla.

\section{Statistical analysis}

The data are presented as means \pm standard deviation (SD) and were analyzed using independent Student's t-test to compare two groups using GraphPad Prism 6.0 software. A value of $p<0.05$ was considered statistically significant.

\section{RESULTS}

miR-558 is down-regulated during the odontogenic differentiation of hPLFs

To investigate the effect of miR-558 on the process of odontogenic differentiation, human periodontal ligament fibroblasts (hPLFs) were treated with osteogenic induction medium for 14 consecutive days to induce odontogenic differentiation. The efficiency of odontogenic differentiation was determined using Alizarin red staining (Figure $1 \mathrm{~A}$ ), and the expression of osteogenic markers, including Osterix and Runx2, were significantly increased in hPLFs at day 14 after osteogenic induction (Figure $1 \mathrm{~B}$ ). Alkaline phosphatase (ALP) activity was also significantly increased, indicating successful odontogenic differentiation (Figure $1 \mathrm{C}$ ). MiR-558 was down-regulated during the odontogenic differentiation of hPLFs in a time-dependent manner (Figure $1 \mathrm{D})$. These results suggest that miR-558 may be involved in the odontogenic differentiation of hPLFs.
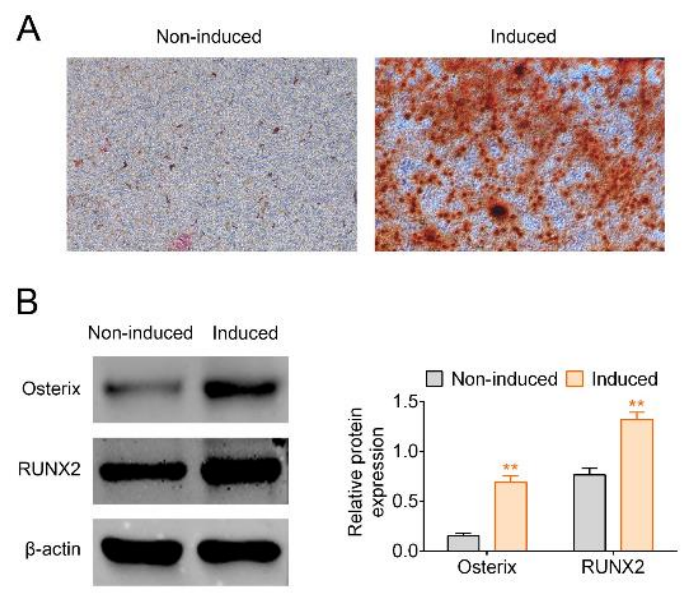

C

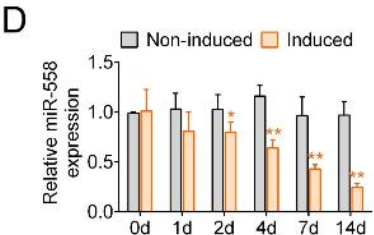

Figure 1: MiR-558 is down-regulated during the odontogenic differentiation of human periodontal ligament fibroblasts (hPLFs). (A) Mineralized nodules were stained using Alizarin red in hPLFs treated with osteogenic induction medium (induced) or vehicle (non-induced) for 14 consecutive days. (B) The protein expression levels of Osterix and Runx2 were determined using Western blotting. (C) ALP activity was measured in the induced and non-induced groups. (D) MiR-558 was measured by qRT-PCR on days $0,1,2,4,7$ and $14 .{ }^{*} P<0.05,{ }^{* *} p<0.01$

\section{MiR-558 inhibits the odontogenic differentiation of hPLFs}

Human periodontal ligament fibroblasts were transfected with the miR-558 mimic or miR-558 inhibitor, and the efficiency was confirmed by qRT-PCR (Figure 2 A). Next, hPLFs were cultured with osteogenic induction medium and treated with the miR-558 mimic or miR-558 inhibitor. Alizarin red staining exhibited fewer mineralized nodules following miR-558 overexpression, but more mineralized nodules were observed in the miR-558 inhibitor group than in the NC group (Figure $2 \mathrm{~B}$ ). Correspondingly, the expression levels of Osterix and Runx2 were significantly decreased in the miR-558 mimic group and significantly increased in the miR-558 inhibitor group compared with those in the NC group (Figure $2 \mathrm{C}$ ). Alkaline 
phosphatase (ALP) activity was significantly decreased following miR-558 overexpression but was significantly increased in the miR-558 inhibitor group compared with that in the NC group (Figure $2 \mathrm{D}$ ). These data indicate that miR558 inhibits the odontogenic differentiation of hPLFs.
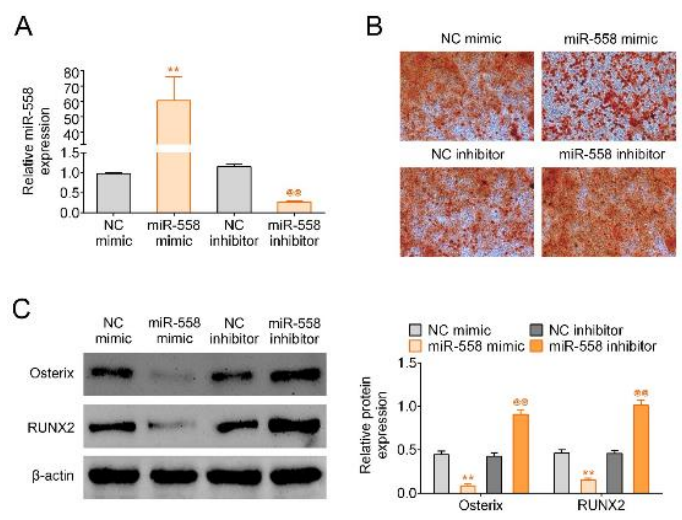

D

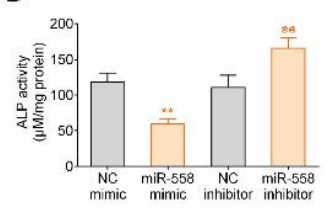

Figure 2: MiR-558 inhibits the odontogenic differentiation of hPLFs. (A) hPLFs were transfected with an miR-558 mimic or an miR-558 inhibitor. (B) Mineralized nodules were stained using Alizarin red in hPLFs with different treatments. (C) The protein levels of Osterix and Runx2 were determined using Western blotting. (D) ALP activity was measured. ${ }^{* *} P<0.01$, $@ @ p<0.01$. * indicates a group versus the NC mimic; $@$ indicates a group versus the NC inhibitor

\section{MiR-558 regulates Notch signaling by targeting Jagged-1}

The Targetscan database (http://targetscan.org/) was used to predict the underlying targets of miR-558, and the complementary sequence of miR-558 was observed in the 3'-UTR of Jagged1 mRNA (Figure $3 \mathrm{~A}$ ). Next, the binding site was mutated and dual-luciferase reporter assays were performed. The luciferase activity of Jagged-1 with the wild-type (WT) 3'-UTR was significantly decreased by treatment in the miR558 mimic group and increased in the miR-558 inhibitor group, whereas the luciferase activity of Jagged-1 with mutant 3'-UTR was not significantly changed (Figure 3 B). Additionally, the mRNA and protein levels of Jagged-1 were significantly decreased in miR-558 mimic group and increased in the miR-558 inhibitor group compared with that in the NC group (Figure $3 \mathrm{C}$ and D). Moreover, downstream targets of Notch signaling, such as HES1 and HEY1, were also examined. The mRNA levels of HES1 and HEY1 were significantly decreased in the miR-558 mimic group and increased in the miR-558 inhibitor group compared with those in the NC group (Figure $3 \mathrm{E}$ ). These data suggest that miR558 regulates Notch signaling by targeting Jagged-1.

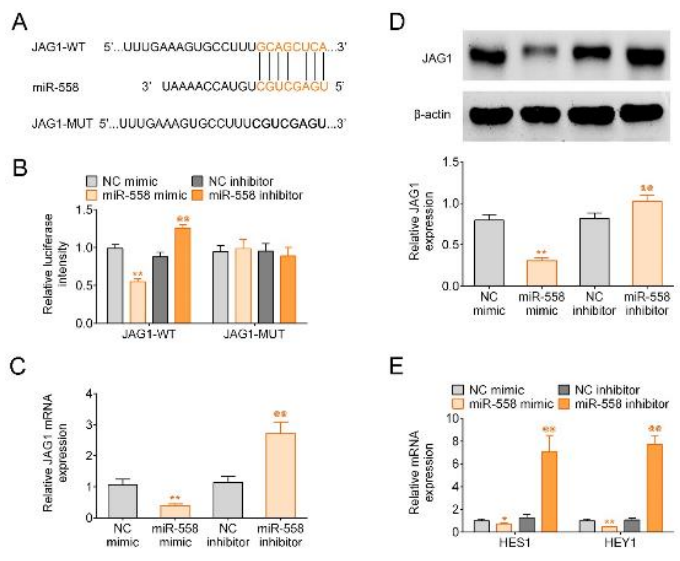

Figure 3: MiR-558 regulates Notch signaling by targeting Jagged-1. (A) The complementary sequence of miR-558 in the 3'-UTR of Jagged-1 mRNA and the mutated binding site. (B) The dual-luciferase reporter assay was performed, and the luciferase activity of Jagged-1 with wild-type (WT) 3'-UTR or mutant (MUT) 3'-UTR was measured. The mRNA (C) and protein (D) levels of Jagged-1 were evaluated in hPLFs treated with the miR-558 mimic or miR-558 inhibitor. (E) The protein levels of HES1 and HEY1 were determined. ${ }^{*} P$ $<0.05,{ }^{* *} p<0.01, @ @<<0.01$. ${ }^{*}$ indicates a group versus the NC mimic; @ indicates a group versus the $\mathrm{NC}$ inhibitor

\section{Jagged-1 knockdown suppresses the odontogenic differentiation of hPLFs}

The mRNA levels of Jagged-1, HES1 and HEY1 were significantly increased when hPLFs were subjected to osteogenic induction medium compared with those in the vehicle groups (Figure $4 \mathrm{~A}$ ). To determine the effect of Jagged-1 during the odontogenic differentiation of hPLFs, Jagged-1 knockdown was performed in hPLFs (Figure $4 \mathrm{~B}$ ). Mineralized nodules were stained using Alizarin red, and fewer mineralized nodules were observed when Jagged-1 was inhibited (Figure $4 \mathrm{C}$ ). The expression levels of Jagged-1, Osterix and Runx2 were significantly decreased in the group with Jagged-1 knockdown compared with those in the NC group (Figure 4 D). Alkaline phosphatase activity was also significantly decreased when Jagged-1 was inhibited (Figure 4 E). Thus, Jagged-1 knockdown suppressed the odontogenic differentiation of hPLFs. Taken together, these data demonstrate that miR-558 inhibits the odontogenic differentiation of hPLFs 
by negatively regulating the Jagged-1/Notch signaling pathway.

A

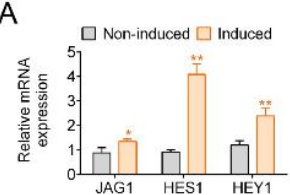

B

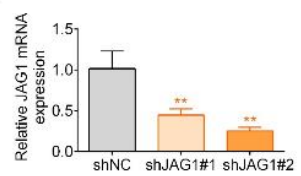

C

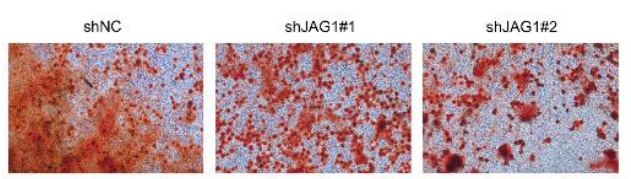

D

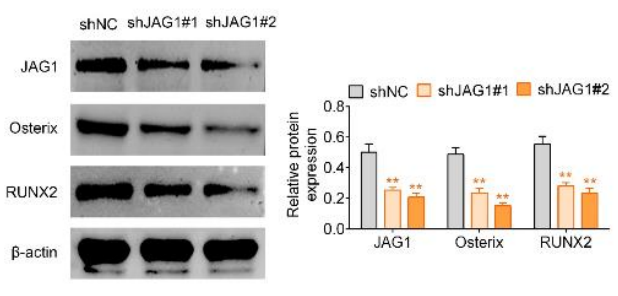

$\mathrm{E}$

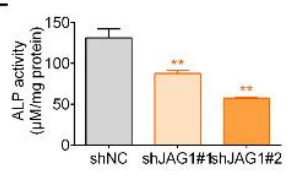

Figure 4: Jagged-1 knockdown suppresses the odontogenic differentiation of hPLFs. (A) The mRNA levels of Jagged-1, HES1 and HEY1 were determined by qRT-PCR. (B) Jagged-1 was knocked down by short hairpin RNA (shRNA). (C) Mineralized nodules were stained using Alizarin red. (D) The expression levels of Jagged-1, Osterix and Runx2 were determined using Western blotting. (E) ALP activity was measured. ${ }^{* \star} P<0.01$

\section{DISCUSSION}

Human periodontal ligament fibroblasts (hPLFs) have the potential of multidirectional differentiation [1]. Under mechanical signals such as bite force and orthodontic treatment, hPLFs tend to differentiate into osteoblasts and participate in periodontal remodeling [2]. Several factors are associated with odontogenic differentiation, and miRNAs regulate odontogenic differentiation [10]. In the current study, we found that miR-558 is down-regulated during the odontogenic differentiation of hPLFs, and miR558 overexpression using an miR-558 mimic inhibits the odontogenic differentiation of hPLFs. Furthermore, the targetscan database was searched to predict the underlying targets of miR-558, and the complementary sequence of miR-558 was observed in 3'-UTR of Jagged-1 mRNA. The molecular mechanism was subsequently explored, and the results suggest that miR-558 regulates Notch signaling by targeting Jagged-1 during the odontogenic differentiation of hPLFs.

Accumulating studies of miR-558 have mainly focused on its role in the tumorigenesis of cancers. For example, miRNA-558 acts as an oncogene in gastric cancer by targeting Smad4 [11]. In addition, miRNA-558 promotes the tumorigenesis of neuroblastoma by activating heparanase [12]. MiRNA-558 is involved in this process by recruiting Argonaute 2 [13]. Moreover, miRNA-558 promotes the radioresistance of A549 cells by targeting apoptosis-associated tyrosine kinase [14]. A previous study also reported that miR-558 regulates cartilage homeostasis by targeting COX-2 [15]. However, the effect of miR-558 on the osteogenic differentiation and underlying mechanism have not yet been reported. In the current study, miR-558 was down-regulated during the odontogenic differentiation of hPLFs, and miR-558 showed an inhibitory effect on the odontogenic differentiation of hPLFs. The current study is the first to show the effect of miR-558 on odontogenic differentiation.

The Notch signaling pathway, as an evolutionarily conservative cellular mechanism, maintains the balance between cell proliferation, differentiation and apoptosis, playing a decisive role in the fate of cell differentiation [16]. The Notch signaling pathway exists widely in vertebrates and invertebrates, and Jagged 1 is its main ligand [17]. Jagged1 activates Notch signaling and promotes the odontogenic differentiation of hPLFs [18]. The targetscan database was searched to predict the underlying targets of miR-558, and Jagged 1 was identified as a potential target of miR-558. Therefore, the Notch signaling pathway may be involved in the odontogenic differentiation of hPLFs in the present study. Further assays suggested that Notch signaling was activated by treatment with an miR-558 inhibitor and downstream targets of Notch signaling, such as HES1 and HEY1, were also significantly increased, demonstrating that miR-558 regulates Notch signaling by targeting Jagged-1 during the odontogenic differentiation of hPLFs. Thus, the molecular mechanism of miR-558 mediating odontogenic differentiation was further clarified in the current study, providing a theoretical basis for its clinical application.

\section{CONCLUSION}

The findings of this study demonstrate the effect of miR-558 on the odontogenic differentiation of human periodontal ligament fibroblasts. Thus, miR-558 may serve as a potential target to 
regulate the odontogenic differentiation of human periodontal ligament fibroblasts.

\section{DECLARATIONS}

\section{Acknowledgement}

Thanks are due to Mr. Amjad Faroog for his technical assistance, and BioMES, Alpha Genomics Private Limited, Islamabad - Pakistan for editing and reviewing the manuscript. Thanks are also due to the patients who participated in the study.

\section{Conflict of interest}

No conflict of interest to disclose regarding this study.

\section{Availability of data and materials}

All the data generated or analyzed during this study are included in this published article.

\section{Contribution of authors}

We declare that this work was performed by the authors named in this article, and all liabilities pertaining to claims related to the content of this article will be borne by the authors. Shengzhang Song designed the study and supervised the data collection. Zhuanjun Yan analyzed and interpreted the data. Wangxi $\mathrm{Wu}$ prepared the manuscript for publication and reviewed the draft of the manuscript. All the authors have read and approved the manuscript.

\section{Open Access}

This is an Open Access article that uses a funding model which does not charge readers or their institutions for access and distributed under the terms of the Creative Commons Attribution License (http://creativecommons.org/licenses/by/ 4.0) and the Budapest Open Access Initiative (http://www.budapestopenaccessinitiative.org/rea d), which permit unrestricted use, distribution, and reproduction in any medium, provided the original work is properly credited.

\section{REFERENCES}

1. de Jong $T$, Bakker $A D$, Everts $V$, Smit $T H$. The intricate anatomy of the periodontal ligament and its development: Lessons for periodontal regeneration. $J$ Periodontal Res 2017; 52(6): 965-974.
2. Smith PC, Martínez C, Martínez J, McCulloch CA. Role of Fibroblast Populations in Periodontal Wound Healing and Tissue Remodeling. Front Physiol 2019; 10: 270.

3. Wahid F, Shehzad A, Khan T, Kim YY. MicroRNAs: synthesis, mechanism, function, and recent clinical trials. Biochim Biophys Acta 2010; 1803(11): 1231-1243.

4. Kabir R, Gupta M, Aggarwal A, Sharma D, Sarin A, Kola MZ. Imperative role of dental pulp stem cells in regenerative therapies: a systematic review. Niger $J$ Surg 2014; 20(1): 1-8.

5. Hu $X$, Zhong $Y$, Kong $Y$, Chen $Y$, Feng J, Zheng J. Lineage-specific exosomes promote the odontogenic differentiation of human dental pulp stem cells (DPSCs) through TGF $\beta 1 /$ smads signaling pathway via transfer of microRNAs. Stem Cell Res Ther 2019; 10(1): 170.

6. Zeng L, Zhao N, Li F, Han D, Liu Y, Liu H, Sun S, Wang $Y$, Feng $H$. miR-675 promotes odontogenic differentiation of human dental pulp cells by epigenetic regulation of DLX3. Exp Cell Res 2018; 367(1): 104111.

7. Liu F, Wang $X$, Yang $Y$, Hu R, Wang $W$, Wang $Y$. The suppressive effects of miR-508-5p on the odontogenic differentiation of human dental pulp stem cells by targeting glycoprotein non-metastatic melanomal protein B. Stem Cell Res Ther 2019; 10(1): 35.

8. Dai $Y$, Wilson $G$, Huang B, Peng $M$, Teng $G$, Zhang $D$, Zhang R, Ebert MP, Chen J, Wong BC et al. Silencing of Jagged1 inhibits cell growth and invasion in colorectal cancer. Cell Death Dis 2014; 5(4): e1170.

9. Zhang Y, Wang J, Liu X, Li J, Fan S. MicroRNA miR103a-3p targets NPAS3 to regulate progression of Alzheimer's disease. Trop J Pharm Res 2020; 19(5): 1015-1021.

10. Gong Q, Wang R, Jiang H, Lin Z, Ling J. Alteration of microRNA expression of human dental pulp cells during odontogenic differentiation. J Endod 2012; 38(10): 13481354.

11. Zheng L, Jiao W, Song $H, Q u H$, Li D, Mei H, Chen $Y$, Yang $\mathrm{F}, \mathrm{Li} H$, Huang $K$ et al. miRNA-558 promotes gastric cancer progression through attenuating Smad4mediated repression of heparanase expression. Cell Death Dis 2016; 7(9): e2382.

12. Qu H, Zheng L, Pu J, Mei H, Xiang X, Zhao X, Li D, Li S, Mao $L$, Huang $K$ et al. miRNA-558 promotes tumorigenesis and aggressiveness of neuroblastoma cells through activating the transcription of heparanase. Hum Mol Genet 2015; 24(9): 2539-2551.

13. Qu $H$, Zheng $L$, Song $H$, Jiao $W$, Li D, Fang $E$, Wang $X$, Mei $\mathrm{H}, \mathrm{Pu} J$, Huang $\mathrm{K}$ et al. microRNA-558 facilitates the expression of hypoxia-inducible factor 2 alpha through binding to 5'-untranslated region in neuroblastoma. Oncotarget 2016; 7(26): 40657-40673.

14. Zhu RX, Song $C H$, Yang JS, Yi QT, Li BJ, Liu SH. Downregulation of AATK mediates microRNA-558induced resistance of A549 cells to radiotherapy. Mol Med Rep 2016; 14(3): 2846-2852.

15. Park SJ, Cheon EJ, Kim HA. MicroRNA-558 regulates the expression of cyclooxygenase-2 and IL-1 $\beta$-induced

Trop J Pharm Res, June 2021; 20(6): 1136 
catabolic effects in human articular chondrocytes. Osteoarthritis Cartilage 2013; 21(7): 981-989.

16. Artavanis-Tsakonas S, Rand MD, Lake RJ. Notch signaling: cell fate control and signal integration in development. Science 1999; 284(5415): 770-776.

17. Bray SJ. Notch signalling: a simple pathway becomes complex. Nat Rev Mol Cell Biol 2006; 7(9): 678-689.
18. Manokawinchoke J, Sumrejkanchanakij $P$, Boonprakong L, Pavasant $P$, Egusa H, Osathanon T. NOTCH2 participates in Jagged1-induced osteogenic differentiation in human periodontal ligament cells. Sci Rep 2020; 10(1): 13329. 